\title{
INFLUENCE OF VISCOUS-ELASTIC PROPERTIES OF A CYLINDRICAL SEALING ELEMENT ON ITS SEALING ABILITY
}

\author{
Elkhan M. ABbasov \\ Institute of Mathematics and Mechanics of the National Academy of Sciences of Azerbaijan, Baku, Azerbaijan \\ e-mail: aelhan@mail.ru
}

KakLik O. Rustamova

Baku Engineering University, Baku, Azerbaijan

e-mail: kaklikrustamova@gmail.com

\section{Aynur O. DARISHOVA}

Azerbaijan University of Architecture and Construction, Baku, Azerbaijan

e-mail: aynur.darishova1991@gmail.com

\begin{abstract}
The stress-strain state of a sealing element in the form of a hollow cylinder is defined with regard to viscous-elastic properties of its material. Based on linear laws of heredity, an analytical formula allowing one to determine the axial load necessary for tightness of the surface of the sealing element and the cylinder wall depending on its physic-mechanical properties and geometrical dimensions is found. Influence of viscous-elastic properties of the material of the sealing element on its sealing ability is realized based on the hypothesis of elastic analogy. The results of numerical calculations are represented in the form of graphs of the external force necessary for achieving sightless. It is shown that viscous-elastic properties of the sealer material greatly influence its sealing ability. Because of heredity of the sealer material, values of the external forces drop in some cases by about five times.
\end{abstract}

Keywords: contact pressure, sealing element, boundary condition, potential energy, functional

\section{Introduction}

In the modern industry, machines and instruments require elastomeric materials like rubber for the purposes of sealing, noise attenuation and shock absorption. The properties of rubber-like elastomers are strongly influenced by the application of mechanical loading, deformation, applied stress-strain rate, temperature, humidity and time. The main characteristic of their behavior is the so-called viscoelastic response. This refers to a simultaneous elastic and viscous response which is more pronounced during creep, relaxation and dynamic mechanical loading of these materials. There is a lot of scientific work devoted to the investigation of behavior of various rubber-like elements, such as sealing elements, O-rings, biodegradable polymers, shock-absorbing elements and in exploitation processes (Dymnikov and Lavendelis, 2002; Gent, 2001; Gonca and Švabs, 2011; Švabs and Gonca, 2012; Švabs, 2013). Elastomers present very complicated mechanical behavior that exceeds the linear elastic theory and contain large deformations, plastic and viscoelastic properties and stress softening (Chagnon et al., 2004; Näser et al., 2005).

It was shown that material compressibility causes additional stresses mostly associated with an additional hydrostatic pressure, and adding a small volume change to classical rubber elastic models can aid analytical and numerical simulations of polymer structures (Cassenti and Staroselsky, 2017). 
Ali et al. (2010) reviewed the needs of different constitutive models for rubber like materials undergoing large elastic deformation.

In some studies, the focus was given to the study of creep and stress relaxation behavior of biodegradable polymers (Guedes et al., 2017; Martins et al., 2015; Singh et al., 2020).

A very important engineering problem is estimating the deformation and stresses in polymer sealers. Sealing elements can be used in demanding seal applications over a broad pressure and temperature range. They are easy to assemble and readily available, but require some attention to prevent application problems. Cylindrical sealers are widely used as seal elements in engineering practice in general, and in oil and gas industry in particular, and their deformation is very important, especially due to the fact that seal breakage could lead to severe natural gas and oil production accidents. Achieving tightness with the least external load by sealers and improving their efficiency and determination of sealing parameters has an important scientific value. Some scholars have done relevant research on cylindrical sealers used in oil and gas production (Lan et al., 2019; Liu et al., 2020).

Liu et al. (2020) carried out theoretical calculation and experimental research through the finite element analysis on the contact with large deformation according to the design of the new seal structure. Lan et al. (2019) investigated the packer rubber with a conventional structure and optimized the materials and the structure of the packer rubber by ABAQUS software simulation and experimental research. But in these works viscoelastic properties of the sealing element were not taken into account.

In consideration of the analysis results of the above scholars and experts, the influence of viscoelastic properties of the cylindrical sealing element on its sealing ability is discussed in this paper. As the experience of using sealing elements shows, the edge effects and heredity have a significant influence on their sealing ability. Therefore, the study of sealing ability of cylindrical sealing elements with regard to edge effects and heredity and the development, on this basis, of effective measures to improve their efficiency has both a practical and scientific value.

In this paper, analytical formulas allowing one to determine the influence of viscous-elastic behavior of the sealing element on its strain state are defined on the basis of linear laws of heredity. We determine relaxation of the axial load necessary for tightness of the surface of the sealing element and the cylinder wall, which is achieved by one-sided axial compression. Accounting for heredity, the magnitude of the axial load for achieving tightness with regard to differences of the strain state before and after contact of the inner surface of the sealing element with the cylinder wall is defined.

\section{Statement of the problem}

Consider a sealing element tightly put on the stock and with a gap $\delta$ between its inner surface and the cylinder wall (Fig. 1). The tightness of the surface of the sealing element and the cylinder wall is achieved by one-sided axial compression (Fig. 1). Assume that the material of the sealing element is homogeneous, then we accept its deformation as axially-symmetric.

We locate the origin of the coordinate system in the center of the lower section of the sealing element, direct the coordinate axis $z$ vertically-upwards, the axis $r$ to the direction of the increasing radius as shown in Fig. 1.

Accounting for viscous-elastic properties of the material of the sealing element on sealing ability may be realized based on the hypothesis of elastic analogy (Abbasov et al., 2020; Lavandelis, 1976; Papanicolaou and Zaoutsos, 2019; Rustamova and Darishova, 2020). By this hypothesis, when passing from elastic calculation to viscous-elastic one, only the dependence between the stresses and strains changes. 


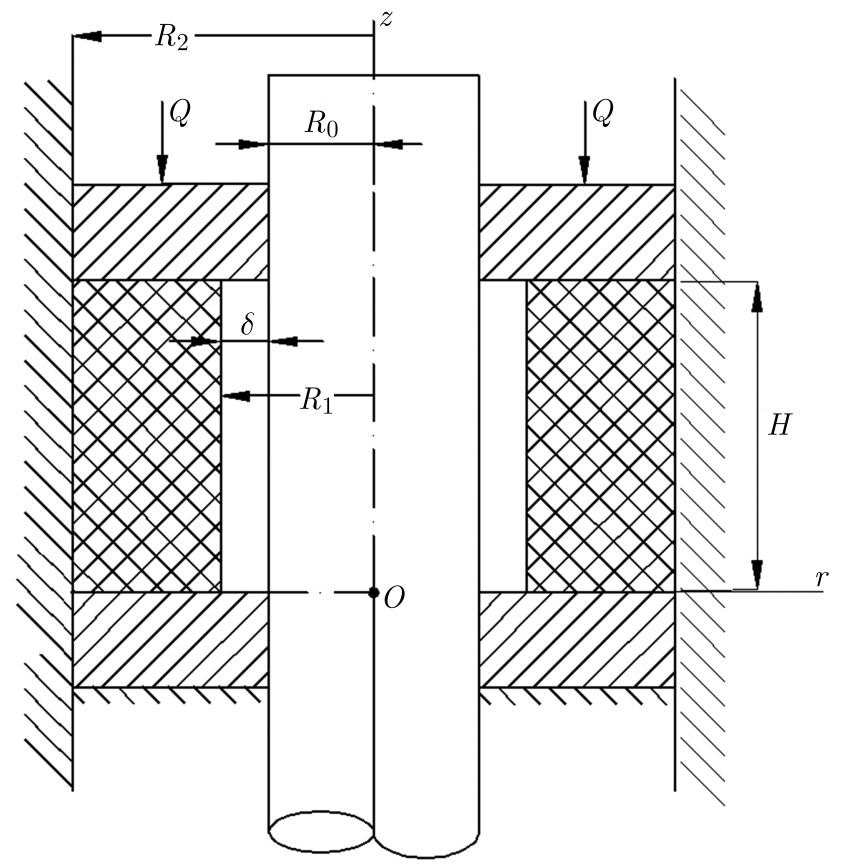

Fig. 1. Design scheme

It should be noted that by the elastic analogy, all stress components satisfy the dependence between stresses and strain obtained on the basis of the chosen model for a uniaxial stress-strain state.

\section{Solution of the problem}

The dependence between the stress-strain components for an arbitrary case of loading in a model that describes best the viscous-elastic behavior of the material of a sealing element is of the form (Lavandelis, 1976)

$$
\dot{\tau}_{i j}+\lambda^{*} \tau_{i j}=G\left[2\left(\dot{\varepsilon}_{i j}+\nu^{*} \varepsilon_{i j}\right)+\delta_{i j}\left(\dot{s}+\nu^{*} s\right)\right]
$$

where $E_{1}=E_{M}, \lambda^{*}=\left(E_{1}+E_{2}\right) / \eta, \nu^{*}=E_{2} / \eta, \eta$ is dynamical viscosity of the material of the sealing element, $E_{M}$ is the instantaneous modulus of elasticity, $E_{2}$ is the elasticity modulus, $G$ is the shear modulus of the sealing element, $\tau_{i j}$ are stress components, $\varepsilon_{i j}$ are relative strain components, $\delta_{i j}$ is Kronecker's symbol, $\dot{\tau}_{i j}$ and $\dot{\varepsilon}_{i j}$ is the time derivative of the stress and strain components, $s$ is dimensionless hydrostatic pressure $s=\left(\sigma_{x}+\sigma_{y}+\sigma_{z}\right) / E$.

Based on the elastic analogy, we represent (Abbasov et al., 2020; Lavandelis, 1976; Papanicolaou and Zaoutsos, 2019; Rustamova and Darishova, 2020)

$$
\varepsilon(\bar{x}, t)=\varepsilon(\bar{x}) \varepsilon(t)
$$

For a slightly compressible material (Lavandelis, 1976)

$$
\varepsilon_{i i}=\frac{3}{2} \frac{1-2 \mu}{1+\mu} s
$$

from which it follows that the nature of these functions is such that they must fulfill the equality

$$
s(t)=\varepsilon(t)
$$


Substituting expression (3.2) into formula (3.1) and considering (3.3), we get

$$
\dot{\tau}_{i j}+\lambda^{*} \tau_{i j}=G\left[2 \varepsilon_{i j}(\bar{x})+\delta_{i j} s(\bar{x})\right]\left[\dot{\varepsilon}_{i j}(t)+\nu^{*} \varepsilon_{i j}(t)\right]
$$

Integrating expression (3.4) with the initial condition $\tau_{i j}(\bar{x}, 0)=G\left[2 \varepsilon_{i j}(\bar{x})+\delta_{i j} s(\bar{x})\right]$, we get

$$
\tau_{i j}=\left[2 \varepsilon_{i j}(\bar{x})+\delta_{i j} s(\bar{x})\right] G\left(\mathrm{e}^{-\lambda^{*} t}+\int_{0}^{t}\left[\dot{\varepsilon}_{i j}(\xi)+\nu^{*} \varepsilon_{i j}(\xi)\right] \mathrm{e}^{-\lambda^{*}(t-\xi)} d \xi\right)
$$

Accepting the elastic analogue idea, introducing the denotation

$$
\bar{G}=G\left(\mathrm{e}^{-\lambda^{*} t}+\int_{0}^{t}\left[\dot{\varepsilon}_{i j}(\xi)+\nu^{*} \varepsilon_{i j}(\xi)\right] \mathrm{e}^{-\lambda^{*}(t-\xi)} d \xi\right)
$$

we can represent expression (3.5) in the form looking like the Hooke law (Lavandelis, 1976)

$$
\tau_{i j}=\bar{G}\left[2 \varepsilon_{i j}(\bar{x})+\delta_{i j} s(\bar{x})\right]
$$

Therefore, in the given case, after finding the solution of the problem in the elastic statement, using expressions (3.6) and (3.7) we can determine stress with regard to hereditary properties of the sealer.

\subsection{Elastic solution}

Abbasov and Rustamova (2015) solved problem in the elastic statement using the methods of elasticity (Amenzadeh, 1976; Lavandelis, 1976). The solution of the problem was performed in two stages in the elastic statement. The first step was compression of the sealing element to the first contact of its outside surface with the cylinder wall, the second state was to achieve tightness.

Let us consider the first stage. Since the material of the sealing element is homogeneous, its deformation is assumed to be axisymmetric. The hypothesis of flat sections can then be used, assuming that the axial deformation of the sealing element depends only on the coordinate in the axial direction.

We locate the origin of the coordinate system in the center of the lower section of the sealing element, direct the coordinate axis $z$ vertically-upwards, the axis $r$ to the direction of the increasing radius (Fig. 1).

Taking into account the above assumptions, the deformation of the sealing element in the axial direction is taken in the form (Abbasov and Rustamova, 2015)

$$
w_{1}=f_{1}(z)
$$

where $f_{1}(z)$ is an unknown function, depending on $z$ and to be determined.

Taking the material of the sealing element to be incompressible (Abbasov and Rustamova, 2015; Amenzadeh, 1976; Gent, 2001; Lavandelis, 1976), we have the equality

$$
\frac{1}{r} \frac{\partial\left(u_{1} r\right)}{\partial r}+\frac{\partial w_{1}}{\partial z}=0
$$

where $u_{1}(r, z)$ is the deformation of the sealing element in the radial direction.

The boundary condition is

$$
\left.u_{1}(r, z)\right|_{r=R_{2}}=0
$$


Then, from expression (3.9), taking into account the boundary condition (3.10), we obtain

$$
u_{1}(r, z)=\frac{1}{2}\left(\frac{R_{2}^{2}}{r}-r\right) f_{1}^{\prime}(z)
$$

For the potential energy of the sealing element after its deformation, taking into account the axisymmetry and linearity, we have the equality (Abbasov and Rustamova, 2015; Lavandelis, 1976)

$$
\Pi=4 \pi G \int_{0}^{H} \int_{R_{1}}^{R_{2}}\left(\varepsilon_{r}^{2}+\varepsilon_{\theta}^{2}+\varepsilon_{z}^{2}+\frac{1}{2} \gamma_{r z}^{2}\right) r d r d z-\int_{0}^{H} Q f_{1}^{\prime}(z) d z
$$

where $H$ is height, $R_{1}, R_{2}$ are inner and outer radii of the sealer, $\mu$ is the friction coefficient between the washer and the end of the sealer, $\varepsilon_{r}, \varepsilon_{\theta}, \varepsilon_{z}$ and $\gamma_{r z}$ are respectively, radial, tangential, axial and shear deformations (Amenzadeh, 1976; Lavandelis, 1976).

The boundary conditions are

$$
\left.\mu Q\right|_{z=H}=\left.2 \pi G \int_{R_{1}}^{R_{2}} \gamma_{z r} r d r \quad w_{1}\right|_{z=0}=\left.0 \quad u_{1}(r, z)\right|_{\substack{z=0 \\ r=R_{1}}}=-\delta
$$

The radial stress at any point of the sealing element can be determined by the formula (Abbasov and Rustamova, 2015; Lavandelis, 1976)

$$
\sigma_{r}=G\left(2 \varepsilon_{r}+s\right)
$$

with the boundary condition

$$
\left.\sigma_{r}\right|_{r=R(z)}=0
$$

where $R(z)=R_{1}+\left.u_{1}(r, z)\right|_{r=R_{1}}$.

Then, from condition (3.15), taking into account expressions (3.11) and (3.14), we obtain

$$
s=\left(\frac{R_{2}^{2}}{R^{2}(z)}+1\right) f_{1}^{\prime}(z)
$$

The axial load $Q$ for compressing the seal is determined by the formula

$$
\left.\pi\left(R_{2}^{2}-R_{1}^{2}\right) \sigma_{z}\right|_{z=H}=Q
$$

On the other hand, we have the equality

$$
\sigma_{z}=G\left(2 \varepsilon_{z}+s\right)
$$

From expression (3.17), taking into account expressions (3.11), (3.13)-(3.16) and (3.18), for the axial load $Q$ for compression of the sealing element to the first contact of its inner surface with the cylinder wall we obtain (Abbasov and Rustamova, 2015)

$$
Q=\pi G\left(R_{2}^{2}-R_{1}^{2}\right) q
$$


where

$$
\begin{aligned}
q= & \sqrt[3]{-\frac{1}{2}\left(\frac{2 a^{3}}{27}-\frac{a b}{3}+c\right)+\sqrt{\frac{1}{4}\left(\frac{2 a^{3}}{27}-\frac{a b}{3}+c\right)^{2}+\left(-\frac{a^{2}}{9}+\frac{b}{3}\right)^{3}}} \\
& +\sqrt[3]{-\frac{1}{2}\left(\frac{2 a^{3}}{27}-\frac{a b}{3}+c\right)-\sqrt{\frac{1}{4}\left(\frac{2 a^{3}}{27}-\frac{a b}{3}+c\right)^{2}+\left(-\frac{a^{2}}{9}+\frac{b}{3}\right)^{3}}}-\frac{a}{3} \\
a= & \frac{1}{\lambda C(3 C-1)}\left[-\frac{3 \delta C}{\cosh (k H)}+2(3 C-1)\left(R_{1}-\frac{\delta}{\cosh (k H)}\right)\right] \\
b= & \frac{1}{\lambda^{2} C^{2}(3 C-1)}\left[R_{2}^{2} C+(3 C-1)\left(R_{1}-\frac{\delta}{\cosh (k H)}\right)^{2}-\frac{6 \delta C}{\cosh (k H)}\left(R_{1}-\frac{\delta}{\cosh (k H)}\right)\right] \\
c= & \frac{-R_{2}^{2}-3\left(R_{1}-\frac{\delta}{\cosh (k H)}\right)^{2}}{\lambda^{3} C^{2}(3 C-1) \cosh (k H)} \delta \\
C= & \frac{\mu\left(R_{2}^{2}-R_{1}^{2}\right) \sinh (k H)}{B k \cosh (k H)}-\frac{\left(R_{2}^{2}-R_{1}^{2}\right)[1-\cosh (k H)]}{4\left(R_{2}^{2}-\frac{3}{2} R_{1}^{2}+\frac{1}{2} \frac{R_{2}^{4}}{R_{1}^{2}}\right) \cosh (k H)} \\
k= & \sqrt{\frac{R_{2}^{2}-\frac{3}{2} R_{1}^{2}+\frac{1}{2} \frac{R_{2}^{4}}{R_{1}^{2}}}{-\frac{3}{64} R_{2}^{4}-\frac{1}{64} R_{1}^{4}+\frac{1}{16} R_{2}^{2} R_{1}^{2}+\frac{1}{16} R_{2}^{4} \ln \frac{R_{2}}{R_{1}}}} \\
B= & \frac{R_{2}^{3}}{3}-\frac{1}{2} R_{2}^{2} R_{1}+\frac{R_{1}^{3}}{6} \\
A= & \frac{q\left(R_{2}^{2}-R_{1}^{2}\right)}{4\left(-\frac{3}{64} R_{2}^{4}-\frac{1}{64} R_{1}^{4}+\frac{1}{16} R_{2}^{2} R_{1}^{2}+\frac{1}{16} R_{2}^{4} \ln \frac{R_{2}}{R_{1}}\right)}
\end{aligned}
$$

Let us also determine the value of the axial load required for full contact of the inner surface of the sealing element with the cylinder wall. The origin of the coordinate system is placed in the center of the lower section of the sealing element and the $z$ axis is directed vertically upward, and the $r$ axis is directed in the direction of the increasing radius.

Using the hypothesis of flat sections and, accordingly, assuming that the axial deformation of the sealing element depends only on the coordinate in the axial direction $z$, we can take

$$
w_{2}=f_{2}(z)
$$

where $w_{2}$ is the axial deformation of the sections of the sealing element, $f_{2}(z)$ is an unknown function depending only on $z$.

The boundary condition is

$$
\left.u_{2}(r, z)\right|_{r=R_{2}}=0
$$

Similarly to the first stage, from the incompressibility condition, we obtain

$$
u_{2}=\frac{1}{2}\left(\frac{R_{2}^{2}}{r}-r\right) f_{2}^{\prime}(z)
$$

For potential energy of the sealing element after its deformation, taking into account the axisymmetry of the problem, we have the equality (Abbasov and Rustamova, 2015; Lavandelis, 1976)

$$
\Pi=4 \pi G \int_{0}^{h} \int_{R_{0}}^{R_{2}}\left(\varepsilon_{r}^{2}+\varepsilon_{\theta}^{2}+\varepsilon_{z}^{2}+\frac{1}{2} \gamma_{r z}^{2}\right) r d r d z-\int_{0}^{h} P f_{2}^{\prime}(z) d z
$$


where $h$ is height of the sealer after deformation of the sealing element to the first contact of its inner surface with the cylinder wall, $R_{0}$ is radius of the cylinder being sealed.

The boundary conditions are

$$
\left.\mu P\right|_{z=h}=\left.2 \pi G \int_{R_{0}}^{R_{2}} \gamma_{z r} r d r \quad u_{2}(r, z)\right|_{\substack{r=R(h) \\ z=h}}=-\left.\delta(h) \quad w_{2}\right|_{z=0}=0
$$

where

$$
R(h)=R_{1}+\left.u_{1}(r, z)\right|_{\substack{r=R_{1} \\ z=h}} \quad \delta(h)=\delta+\left.u_{1}(r, z)\right|_{\substack{r=R_{1} \\ z=h}}
$$

The axial force required to deform the sealing element until its outer surface is in full contact with the cylinder wall is determined by the formula

$$
\left.\pi\left(R_{2}^{2}-R_{0}^{2}\right) \sigma_{z}\right|_{z=h}=P
$$

where $\sigma_{z}$ is the axial stress in any cross-section of the sealing element.

After full contact of the outer surface of the sealing element with the cylinder wall, the boundary condition on the upper section has the form

$$
\left.\sigma_{r}\right|_{\substack{r=R_{0} \\ z=h}}=0
$$

Then, from expression (3.14), taking into account boundary condition (3.26), we obtain

$$
s=\left(\frac{R_{2}^{2}}{R_{0}^{2}}+1\right) f_{2}^{\prime}(h)
$$

From expression (3.25), for the axial load $P$ necessary for complete contact of the inner surface of the sealing element with the cylinder wall we have (Abbasov and Rustamova, 2015)

$$
P=2 \pi G\left(R_{2}^{2}-R_{0}^{2}\right) \frac{R(h) \delta(h)}{R_{2}^{2}-R^{2}(h)}\left(\frac{R_{2}^{2}}{\left(R_{0}^{2}\right.}+3\right)
$$

where

$$
\begin{aligned}
& R(h)=R_{1}+\lambda\left(c_{2} \sinh (k h)+c_{3} \cosh (k h)+\frac{A}{k^{2}}\right) \\
& \delta(h)=\delta+\lambda\left(c_{2} \sinh (k h)+c_{3} \cosh (k h)+\frac{A}{k^{2}}\right) \\
& c_{2}=\frac{\mu Q}{\pi G B k \cosh (k H)}+\left(\frac{\delta}{\lambda}+\frac{A}{k^{2}}\right) \tanh (k H) \quad c_{3}=-\frac{\delta}{\lambda}-\frac{A}{k^{2}} \\
& c_{4}=-\frac{\mu q\left(R_{2}^{2}-R_{1}^{2}\right)}{B k^{2} \cosh (k H)}-\left(\frac{\delta}{\lambda k}+\frac{A}{k^{3}}\right) \tanh (k H) \\
& h=H-\left|\frac{c_{2}}{k} \cosh (k H)+\frac{c_{3}}{k} \sinh (k H)+\frac{A}{k^{2}} H+c_{4}\right|
\end{aligned}
$$

The contact pressure between the inner surface of the sealing element and the cylinder wall after their full contact can be determined (by analogy to the beam on an elastic foundation), according to the formula

$$
\sigma_{r}(z)=k_{0} u_{0}(z)
$$


where $k_{0}$ is the bed ratio

$$
u_{0}(z)=R_{1}-R_{0}+\left.u_{1}(r, z)\right|_{r=R_{1}}
$$

We now consider the case when the sealing element deforms uniformly. Based on the elastic analogy (Lavandelis, 1976; Papanicolaou and Zaoutsos, 2019), we accept the deformation of cross sections of the sealer in the form (Fig. 2)

$$
\varepsilon_{1}(z, t)=\varepsilon_{1}(z) \varepsilon_{1}(t)
$$

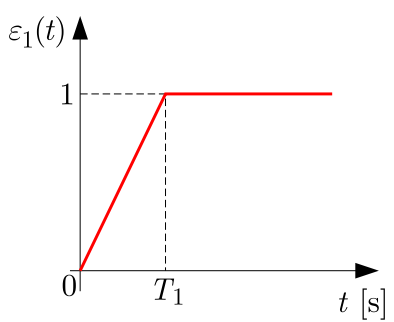

Fig. 2. Graph of the time dependence of the relative axial deformation of the upper section

Now consider how the stress $\sigma_{z}(Q)$ at the end surfaces will change over time (relaxation) if the sealing element is deformed at a steady rate until a fixed time $T_{1}$ and then it stays stable

$$
\varepsilon_{1}(t)=\frac{t}{T_{1}}\left[H(t)-H\left(t-T_{1}\right)\right]+H\left(t-T_{1}\right)
$$

where $H(t)$ is the Heaviside function, $T_{1}$ is time of deformation of the upper section of the sealing element to its first contact of the inner surface with the cylinder wall.

Substituting expression (3.31) into formula (3.6) and then integrating it, we obtain the equality

$$
\begin{gathered}
\bar{G}\left(t, T_{1}\right)=G\left\{\mathrm{e}^{-\lambda^{*} t}+\left[\left(\left(\nu^{*}-\lambda^{*}\right)\left(1-\mathrm{e}^{-\lambda^{*}\left(t-T_{1}\right)}\right)-\nu^{*} \lambda^{*}\left(t-T_{1}\right)\right) H\left(t-T_{1}\right)\right.\right. \\
\left.\left.-\left(\left(\nu^{*}-\lambda^{*}\right)\left(1-\mathrm{e}^{-\lambda^{*} t}\right)-\nu^{*} \lambda^{*} t\right) H(t)\right]\right\}
\end{gathered}
$$

Then, allowing for formula (3.32) following from expression (3.19), we get

$$
Q=\pi \bar{G}\left(t, T_{1}\right)\left(R_{2}^{2}-R_{1}^{2}\right) q
$$

Expression (3.33) formulates the magnitude of the axial load necessary for recompressing the sealing element to its first contact of its inner surface with the cylinder wall.

Substituting formulas (3.32) into expression (3.28) for the axial load necessary for full contact of the inner surface of the sealing element with the cylinder wall with regard to heredity, we get

$$
P=2 \pi \bar{G}\left(t, T_{2}\right)\left(R_{2}^{2}-R_{0}^{2}\right) \frac{R(h) \delta(h)}{R_{2}^{2}-R^{2}(h)}\left(\frac{R_{2}^{2}}{\left(R_{0}^{2}\right.}+3\right)
$$

where $T_{2}$ is time of deformation of the upper section of the sealing element to its full contact of the inner surface with the cylinder wall.

The axial load for compressing the sealing element until its inner surface is in full contact with the cylinder wall in the first approximation is the sum of the axial loads of the first and second stages

$$
P^{*}=Q+P
$$




\section{Numerical calculation}

Thus, based on theoretical investigations, the analytical formulas allowing one to determine the axial loads necessary for the first and full contact of the inner surface of the sealing element with the cylinder wall depending on its physic-mechanical properties and geometrical dimensions are found.

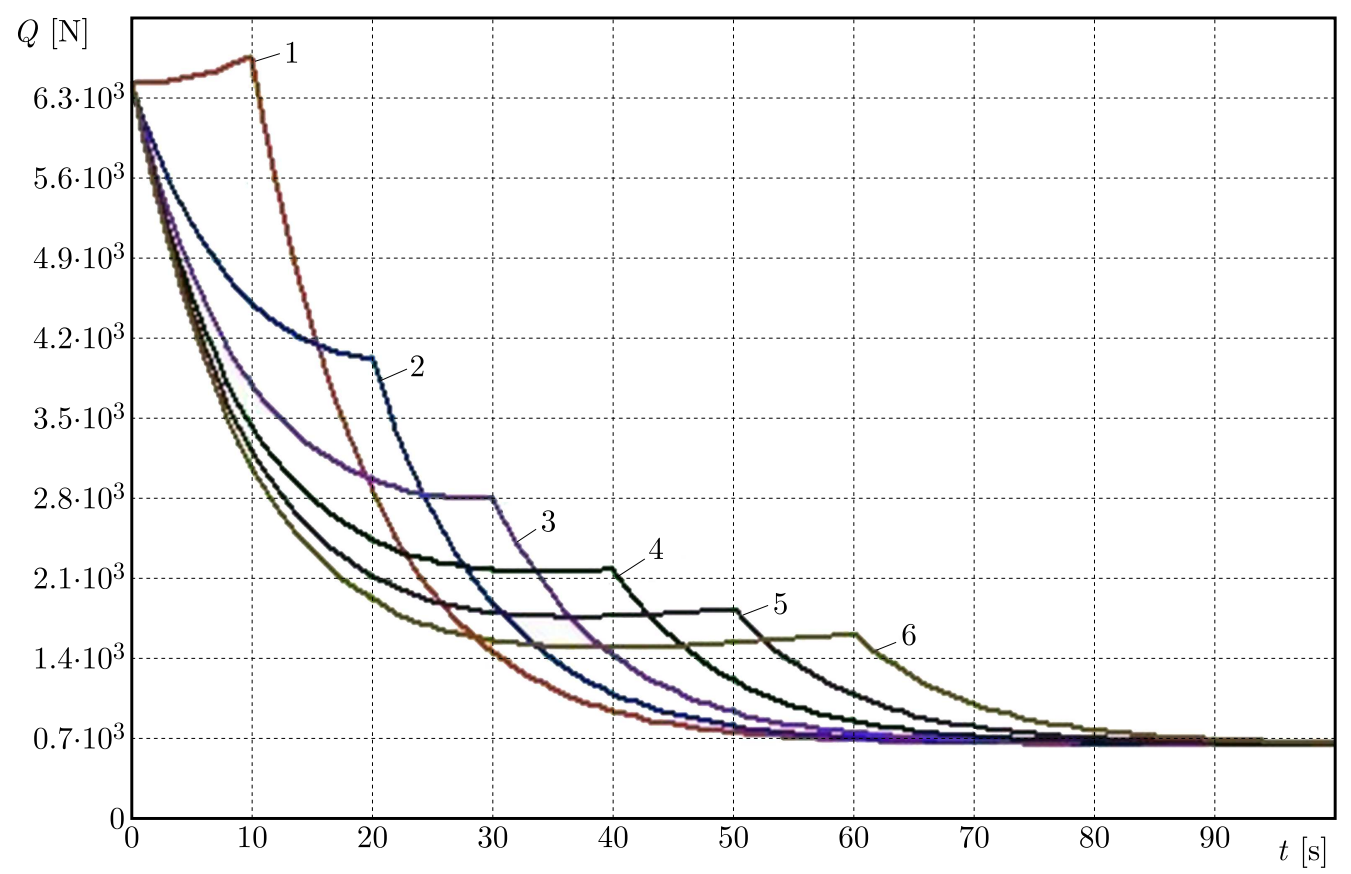

Fig. 3. The graph relaxation of axial stress of the upper section of the sealer to the first contact of its inner surface with the cylinder wall; $1-T_{1}=10 \mathrm{~s}, 2-T_{1}=20 \mathrm{~s}, 3-T_{1}=30 \mathrm{~s}, 4-T_{1}=40 \mathrm{~s}$, $5-T_{1}=50 \mathrm{~s}, 6-T_{1}=60 \mathrm{~s}$

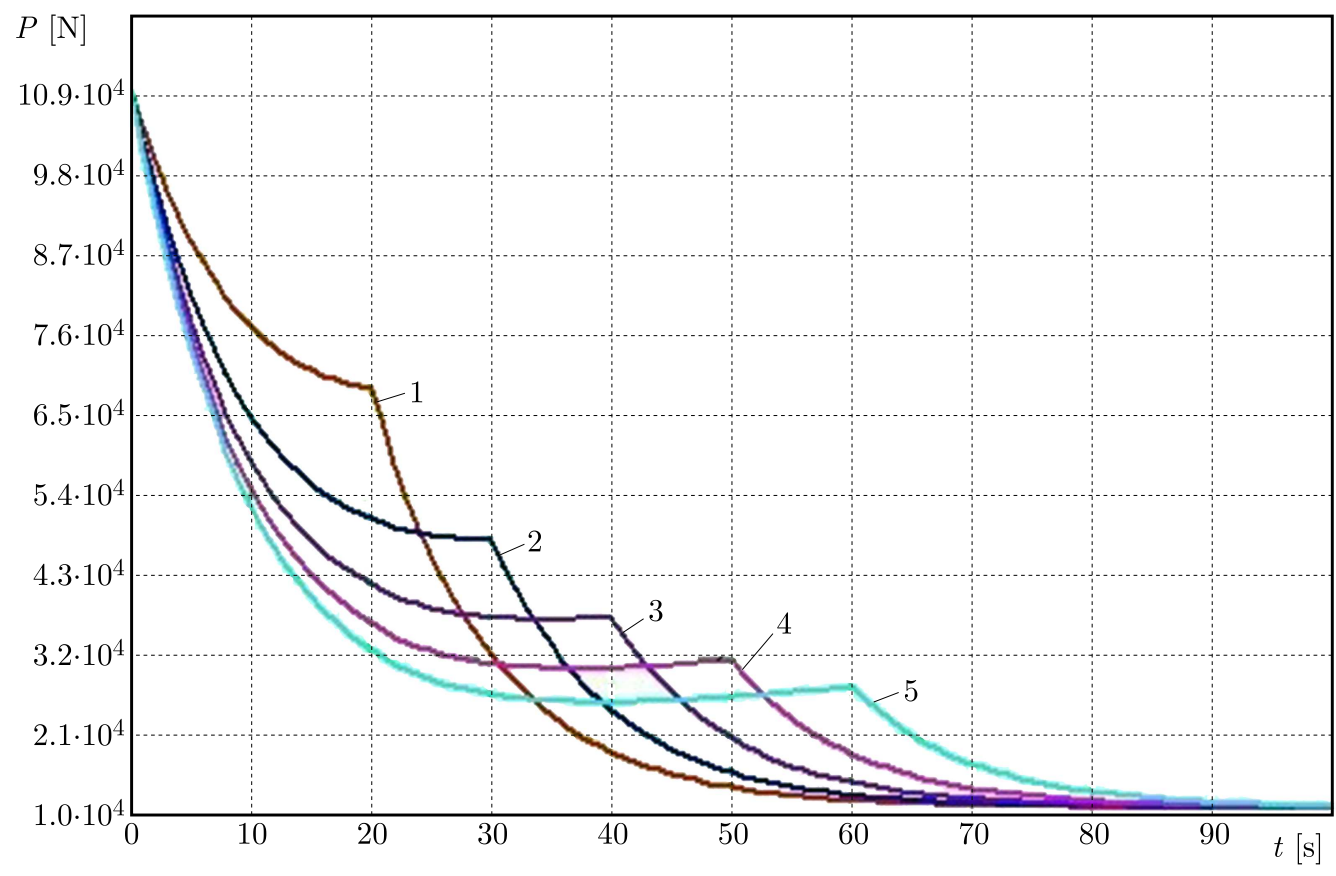

Fig. 4. The graph of relaxation of the axial stress of the upper section of the sealer after full contact of its inner surface with the cylinder wall; $1-T_{2}=20 \mathrm{~s}, 2-T_{2}=30 \mathrm{~s}, 3-T_{2}=40 \mathrm{~s}, 4-T_{2}=50 \mathrm{~s}$, $5-T_{2}=60 \mathrm{~s}$ 
The numerical calculation is made by formulas (3.33) and (3.34) for the values of parameters which are shown in Table 1. The results of numerical calculations are represented in the form of graphs of external forces necessary for achieving sightless (Figs. 3 and 4).

The time dependence of the axial load $Q$ for compression of the sealing element to the first contact of its inner surface with the cylinder wall is depicted in Fig. 3. And Fig. 4 shows the time dependence of the axial load $P$ necessary for complete contact of the inner surface of the sealing element with the cylinder wall.

The same picture is observed when deformation of the sealer happens uniformly. As seen in Figs. 3 and 4, for a constant value of axial deformation in the section of application of the external force with regard to heredity, the stress greatly relaxes. This time, the axial stress relaxation for different velocities of deformation occurs differently (Figs. 3 and 4). As can be seen in the figures, the value of external forces decreases and stabilizes after a while. Because of heredity of the sealer material, the values of external forces in some cases drop by about five times.

It is shown that viscous-elastic properties of the sealer material greatly influence its sealing ability.

Table 1. The values of parameters

\begin{tabular}{|l|c|}
\hline \multicolumn{1}{|c|}{ Variable } & Value \\
\hline \hline$R_{0}$-radius of cylinder being sealed $[\mathrm{m}]$ & 0.073 \\
\hline$R_{1}$ - inner radius of sealer $[\mathrm{m}]$ & 0.076 \\
\hline$R_{2}$ - outer radius of sealer $[\mathrm{m}]$ & 0.1 \\
\hline $\begin{array}{l}\delta-\text { size of gap of abutting inner surface to } \\
\text { wall of cylinder being sealed }[\mathrm{m}]\end{array}$ & 0.003 \\
\hline$H-$ height of sealer [m] & 0.02 \\
\hline$G-$ shear modulus of sealing material [Pa] & $1.3 \cdot 10^{8}$ \\
\hline$\mu-$ friction coefficient between washer and end of sealer $[-]$ & 0.5 \\
\hline$\nu-$ Poisson's ratio $[-]$ & 0.25 \\
\hline$\nu^{*}=E_{2} / \eta[-]$ & 0.01 \\
\hline$\lambda^{*}=\left(E_{1}+E_{2}\right) / \eta[-]$ & 0.1 \\
\hline $\begin{array}{l}T_{1}-\text { time of deformation of upper section of sealing } \\
\text { element to its first contact of inner surface with } \\
\text { cylinder wall [s] }\end{array}$ & $10,15,20,25,30,35$ \\
\hline $\begin{array}{l}T_{2}-\text { time of deformation of upper section of sealing } \\
\text { element to its complete contact of inner surface with } \\
\text { cylinder wall [s] }\end{array}$ & $15,20,25,30,35$ \\
\hline
\end{tabular}

\section{Conclusion}

Thus, it is shown that viscoelastic properties of the sealing material greatly influence its sealing ability and their ignorance may lead to incorrect conclusions. Based on the hypothesis of elastic analogy, an expression is obtained allowing one to determine the stress-strain state of the sealing element in the form of a hollow cylinder with regard to viscous-elastic properties of its material. Relaxation of the axial load necessary for the first and full contact of the inner surface of the sealing element with the cylinder wall is determined. The results of numerical calculations are represented in the form of graphs of the external force necessary for achieving sightless. 


\section{References}

1. Abbasov E.M., Rustamova K.O., 2015, Distribution of contact pressure between a sealing element and a cylinder, International Applied Mechanics, 51, 5, 591-600

2. Abbasov E.M., Rustamova K.O., Darishova A.O., 2020, Contact pressure between the outside surface of the sealer and cylinder wall, Journal of Applied Mathematics and Physics, 8, 2, 349-366

3. Ali A., Hosseini M., Sahari B.B., 2010, A review of constitutive models for rubber-like materials, American Journal of Engineering and Applied Sciences, 3, 1, 232-239

4. Amenzadeh Yu.A., 1976, Theory of Elasticity, Press Vysshaya Shkola, Moscow

5. Cassenti B.N., Staroselsky A., 2017, Deformation and stability of compressible rubber O-rings, International Journal of Mechanical and Materials Engineering, 12, 5

6. Chagnon G., Marckmann G., Verron E., 2004, A comparison of the Hart-Smith model with Arruda-Boyce and Gent formulations for rubber elasticity, Rubber Chemistry and Technology, 77, $724-735$

7. Dymnikov S.I., Lavendelis E.E., 2002, Calculations of rigidity of rubber elastic elements of arched and conical rubber-metal shock absorbers, Scientific Proceedings of Riga Technical University. Series 6: Transport and Engineering (Mechanics), Riga, 164-169

8. Gent A.N., 2001, Engineering with Rubber, Hanser

9. Gonca V., Švabs J., 2011, Definition of Poisson's ratio of elastomers, Proceedings of 10th International Scientific Conference "Engineering for Rural Development", Latvia, Jelgava, 428-434

10. Guedes R.M., Singh A., Pinto V., 2017, Viscoelastic modeling of creep and stress relaxation behavior in PLA-PCL fibers, Fibers and Polymers, 18, 2443-2453

11. LAn W.-J., Wang H.-X., Zhang X., Chen S.-S., 2019, Sealing properties and structure optimization of packer rubber under high pressure and high temperature, Petroleum Science, 16, 3, $632-644$

12. Lavandelis E.E., 1976, Calculation of Rubber Technical Goods, Mashinostroyeniye, Moscow

13. Liu Y., Lian Z., Chen J., Kuang S., Mou Y., Wang Y., 2020, Design and experimental research on sealing structure for a retrievable packer, Shock and Vibration, https://doi.org/10.1155/2020/7695276

14. Martins C., Pinto V., Guedes R.M., Marques A.T., 2015, Creep and stress relaxation behavior of PLA-PCL fibres - A linear modeling approach, Procedia Engineering, 114, 768-775

15. NÄser B., Kaliske M., André M., 2005, Durability simulations of elastomeric structures, [In:] Constitutive Models for Rubber IV, P.E. Austrell and L. Kari (Edit.), AA Balkema Publishers, UK, 45-50

16. Papanicolaou G.C., Zaoutsos S.P., 2019, Viscoelastic constitutive modeling of creep and stress relaxation in polymers and polymer matrix composites, [In:] Creep and Fatigue in Polymer Matrix Composites (2nd Ed.), R.M. Guedes (Edit.), Woodhead Publishing. 3-59

17. Rustamova K.O., Darishova A.O., 2020, Stress relaxation behavior of the annular sealing element - A linear modeling approach, Journal of Contemporary Applied Mathematics, 10, 1, $24-37$

18. Singh A., Guedes R.M., Paiva D., Magalhaes F.D., 2020, Experiment and modelling of the strain-rate-dependent response during in vitro degradation of PLA fibres, SN Applied Sciences, 2,2

19. Švabs J., 2013, The methods of spatial rubber technical products optimal synthesis problems solution, Summary of Thesis of Candidate for a doctor's degree in the program "Mechanical Engineering", Riga 
20. Švabs J., Gonca V., 2012a, Regularization of the boundary value problems for incompressible material, Scientific Works of Riga Technical University. Mechanical Engineering. Nanotechnology. Composite and Rubber Materials, 77-81

21. Švabs J., Gonca V., 2012b, Thin rubber-metal element's stiffness at compression, Scientific Works of Riga Technical University. Mechanical Engineering. Nanotechnology. Composite and Rubber Materials, 87-90

Manuscript received December 29, 2019; accepted for print June 11, 2021 